\title{
Development and in Vitro-in Vivo Evaluation of Controlled Release Matrix Tablets of Desvenlafaxine
}

\author{
Shashidhar Reddy Dodda ${ }^{1}$, Prakash Rao Boggrapu ${ }^{2 *}$ \\ ${ }^{1}$ Department of Pharmacology, Bioneeds Preclinical Services, Bangalore, India; ${ }^{2}$ Department of Pharmaceutics, Karnataka College of \\ Pharmacy, Bangalore, India. \\ Email: "bprao_1111@rediffmail.com
}

Received October1 $14^{\text {th }}, 2011$; revised November $20^{\text {th }}, 2011$; accepted December $16^{\text {th }}, 2011$

\begin{abstract}
The objective of this investigation was to prepare extended release tablet containing matrix granules of Desvenlafaxine succinate monohydrate and to study its in vitro release and in vivo absorption. The design of dosage form was performed by choosing hydrophilic hydroxypropyl methyl cellulose (HPMC K100M), sodium carboxyl methyl cellulose (Blanose), microcrystalline cellulose (MCC) and lactose monohydrate polymers as matrix builders and polyvinyl pyrolidine (Kollidon K30) as granulating polymers. Granules were prepared by composing drug with HPMC K100M, sodium CMC, MCC and lactose monohydrate by spray drying method. Optimized formulation of Desvenlafaxine succinate monohydrate was formed by using 20\% HPMC K100M, 26.6\% MCC, $6.6 \%$ of sodium CMC (Blanose), $13.3 \%$ of lactose monohydrate and 5\% ratio of Kollidon K30 as binder. Tablets were compressed with free flowing optimized granules of uniform drug content. This extended the release period up to $24 \mathrm{~h}$ in vitro study. Similarity factor and mean dissolution time were also reported to compare various dissolution profiles. The network formed by HPMC, MCC and Blanose had been coupled satisfactorily with the controlled resistance. Biopharmaceutical study of this optimized dosage form in rabbit model showed $24 \mathrm{~h}$ prolonged drug release in vivo. A close correlation $\left(\mathrm{R}^{2}=0.9833\right)$ was established between the in vitro release and the in vivo absorption of drug. The results suggested that wet granulation with spray dried technique, is a suitable method to formulate sustained release Desvenlafaxine succinate monohydrate and it can Perform therapeutically better than conventional immediate release dosage form.
\end{abstract}

Keywords: Desvenlafaxine; Pharmacokinetics; ER Matrix Tablet; In Vitro Kinetics; Depression

\section{Introduction}

Developing oral-sustained release formulations for highly water-soluble drugs with constant rate of release has become a challenge to the pharmaceutical technologists. Fast release drug generally causes toxicity if not formulated as extended release dosage form. Among various formulation approaches, in controlling the release of water-soluble drugs, the development of sustained release coated granules has a unique advantage of lessening the chance of dose dumping which is a major problem when highly water-soluble drug is formulated as matrix tablets. Most of the researchers have worked on matrix tablets and multilayered matrix tablets. In the present study, a sustained release dosage form of Desvenlafaxine (DV) has been developed that enables less frequent administering of drug [1-3]. Matrix granules of Desvenlafaxine were formed by appropriate combination of HPMC and sodium $\mathrm{CMC}$, lactose monohydrate, $\mathrm{MCC}$ and Kollidon $\mathrm{K} 30$ was chosen to form the granules of extend duration

"Corresponding author. of drug release.

Desvenlafaxine (o-desmethyl venlafaxine) is an active metabolite of Venlafaxine. Inhibits the neuronal uptake of nor epinephrine, serotonin and to a lesser extent dopamine but have no monoamine oxidase inhibitory activity and low affinity for brain muscaranic, cholinergic, histaminergic or alpha adrenergic receptors [4]. The solubility of Desvenlafaxine is highly dependent on $\mathrm{pH}$; the significant $\mathrm{pH}$ dependency of solubility percents challenges the development of controlled release formulations of Desvenlafaxine for obtaining consistent dissolution profiles $[5,6]$.

The objectives of this work were: 1) to evaluate the physical characters of prepared Tablets 2) to elucidate the effect of polymer composition and the release kinetics, 3) in vivo study.

\section{Materials and Methods}

\subsection{Materials}

Desvenlafaxine succinctness monohydrate was obtained 
from Dr Reddy Ltd, Hyderabad, India.Hypermellose (Methocel K100M CR), Blanose (M31XFPH) were obtained as a gift sample from ColorconAsiaPvt Ltd. Kollidon K30 and Microcrystalline cellulose (Avicell) was obtained from RachemPharmaPvt Ltd. Hyderabad. All other solvents, Reagents and Excipient were purchased from local vendors, India, and were of analytical grade.

\subsection{Drug Excipient Interactions}

The Possibility of Drug Excipient interaction was investigated by differential scanning calorimeter (DSC). The DSC Thermo gram of pure drug and Drug Excipient mixtures were recorded. The samples were separately sealed in aluminum cells and set in Metler TA 4000 Thermal analyzer.

\subsection{Formulation}

Different batches (DVF1, DVF2, DVF3, DVF4, DVF5, DVF6 and DVF7) were prepared according to the Table 1. Desvenlafaxine, Methocel K100M, Blanose and MCC and lactose monohydrate (passed through 40\#) were mixed uniformly in polybag and granulated with aqueous solution of Kollidon K30 followed by spray drying at $60^{\circ} \mathrm{C}$, he dried granules passed through $\# 24$ mesh and finally blend with Talc and magnesium state appropriate quantity and compressed with $9 \mathrm{~mm}$ round standard concave punches.

\subsection{Physical Characterization of Tablets}

Formulated tablets were subjected to different physical characterization studies. The weight variation was determined on 20 tablets using an electronic balance (Sartorius, Germany). Tablet hardness was determined for a minimum of six tablets using a Monsanto (Standard type, Cambridge, UK) tablet hardness tester. Ten tablets were taken and their thickness was recorded by a digital caliper (Mitutoyo, Kawasaki, Japan). Friability was calcu- lated as the percentage weight loss of 20 tablets after Rotation using a Roche friabilator (Basel, Switzerland) for $4 \mathrm{~min}$ at $25 \mathrm{rpm}$.

\subsection{Drug Content of Tablets}

The drug content of the formulated tablets was estimated spectrophotometrically (Shimadzu UV-160A) after extraction in the methanol. Ten tablets were finely powdered; powder sample equivalent to $10 \mathrm{mg}$ of Desvenlafaxine was accurately weighed and transferred to a 100 $\mathrm{mL}$ volumetric flask. Initially $50 \mathrm{~mL}$ of methanol was added to the volumetric flask and allowed to stand for $6 \mathrm{~h}$ with intermittent sonication to ensure complete solubility of the drug. Then the volume was made up with methanol and the mixture was centrifuged. The supernatant liquid was filtered through a $0.2-\mu \mathrm{m}$-membrane filter and Desvenlafaxine was analyzed spectrophotometrically at a wavelength of $240 \mathrm{~nm}$.

\subsection{In Vitro Dissolution Studies}

The in vitro dissolution studies were performed by USP30 type I dissolution apparatus at $100 \mathrm{rpm}$. The dissolution medium consisted of $0.9 \%$ Normal saline and the medium was maintained at $37^{\circ} \mathrm{C} \pm 0.5^{\circ} \mathrm{C}$. An aliquot (5 $\mathrm{ml}$ ) was withdrawn at specific time intervals and replaced with the same volume of fresh medium at same temperature. The withdrawal sample was filtered through $0.45 \mu \mathrm{m}$ filter paper. Next, its drug content was determined by UV-visible spectrophotometer (Shimadzu, Kyoto, Japan) at $240 \mathrm{~nm}$. It was ascertained that none of the ingredients used in the matrix formulations interfered with the assay of drug (DV). The release studies were conducted in triplicate. Mean percent cumulative drug release was plotted against time of release.

\subsection{Kinetics and Mechanism of Drug Release}

Kinetics of drug release was determined by fitting data to

Table 1. Composition of extended release matrix tablet of Desvenlafaxine succinate monohydrate.

\begin{tabular}{|c|c|c|c|c|c|c|c|}
\hline Ingredients (mg) & DVF1 & DVF2 & DVF3 & DVF4 & DVF5 & DVF6 & DVF7 \\
\hline Desvenlafaxine succinate Monohydrate & 76 & 76 & 76 & 76 & 76 & 76 & 76 \\
\hline Hypromellose (Methocel K15 MCR) & 30 & - & 20 & 30 & 40 & 30 & 10 \\
\hline Blanose (M31XFPH) & - & 30 & 10 & 10 & 20 & 15 & 30 \\
\hline Polyvinyl pyrrolidone (Kollidon K30) & 15 & 15 & 15 & 15 & 15 & 15 & 15 \\
\hline Lactose monohydrate & 50 & 50 & 50 & 50 & 50 & 50 & 50 \\
\hline MCC (Avicel) & 120 & 120 & 120 & 110 & 90 & 105 & 110 \\
\hline Purified water & q.s & q.s & q.s & q.s & q.s & q.s & q.s \\
\hline Talc & 6 & 6 & 6 & 6 & 6 & 6 & 6 \\
\hline
\end{tabular}


different equations such as zero order $(\mathrm{M}=\mathrm{kt})$, first order equation $\left(M=\ln M_{0}+k t\right)$, Higuchi model $(M=k \sqrt{ })$ and KorsemeyerPeppas equation $(M=k t n)$. A value of $n$ $=0.5$ indicates case I (Fickian) diffusion, $0.5<\mathrm{n}<1$ is for anomalous (non-Fickian) diffusion, $\mathrm{n}=1$ is for case II transport and $\mathrm{n}>1$ indicates supercase II transport. M is the amount of drug (\%) released after time $t ; \mathrm{M}_{0}$ is the amount of drug released at zero time; $\mathrm{k}$ is the release rate constant and $\mathrm{n}$ is the exponent. Drug release following particular mechanism is judged by the linearity (R2) of plot [7].

\subsection{Stability Studies}

Stability studies were conducted on SR Tablets of select batch to assess their stability with respect to their physical appearance, drug content and release characteristics after storing at $25^{\circ} \mathrm{C}$ under $60 \%$ relative humidity (RH) and $40^{\circ} \mathrm{C}$ under $75 \% \mathrm{RH}$ for 6 months [8].

\subsection{Pharmacokinetic Evaluation}

All animal studies were performed according to the Guidelines for the Care and Use of Laboratory Animals that was approved by the Committee of Ethics of Animal Experimentation of Bioneeds. Extended Release Tablets with $50 \mathrm{mg}$ equivalent wt of Desvenlafaxine and $50 \mathrm{mg}$ equivalent wt of Desvenlafaxine suspension was administer to two groups $(\mathrm{N}=3)$ orally via silicone rubber gastric intubation tube to male albino Rabbits with average weight of $2.5 \mathrm{~kg}$ which were housed individually in standard cage in a room with air, humidity and temperature control. The rabbit's mouth was opened, and wooden rod was inserted between the jaws. A gastric tube was centrally placed to the hole (21.22). Tablets were set on the tip of a gastric intubation tube, and administered in to the stomach of rabbit [9]. After receiving the oral dose, 5 $\mathrm{ml}$ of water was administered to facilitate the accession of the tablets. Rabbits were fasted over night but had free access to water; $2 \mathrm{ml}$ of blood samples were withdrawn from heparin zed branule (G22, G24) placed in the marginal ear vein [10], at each of the pre determined times after administration: $0.25,0.5,1,2,4,6,8$ and $24 \mathrm{hr}$. samples were transferred to $2 \mathrm{ml} \mathrm{K} 2$ EDTA coated tubes and centrifuged at $3000 \mathrm{rpm}$ for $10 \mathrm{~min}$. supernatant plasma was separated and transferred in to 96 well plate and stored at $-20^{\circ} \mathrm{C}$ until Analyzed.

\subsection{In Vivo Data Analysis}

The maximum plasma concentration $\left(\mathrm{C}_{\max }\right)$ and the time to reach the maximum concentration $\left(\mathrm{t}_{\max }\right)$ were directly obtained from the observed values. The area under the curve up to $24 \mathrm{~h}$ after administration (AUC) was calculated by the trapezoidal rule from the observed values.

\section{Results and Discussion}

In the present study, granules were prepared initially using various compositions (Table 1) of matrix forming polymers (HPMC, sodium CMC and MCC) with the help of granulating agent, PVP was used as Binder. Tablets were tested to check dissolution period up to $100 \%$ cumulative drug release. Prolonged release of drug was in the order of DVF3 $<$ DVF2 $<$ DVF $1<$ DVF6 $<$ DVF7 $<$ DVF5 tablets with code no DVF5 exhibitedextended drug release up to $24 \mathrm{hr}$ (Figure 1). Rate of drug release was significant $(p<0.005)$ when combination of HPMC and Blanose as a Matrix builder. Suitable combination of HPMC with sodium CMC and MCC gave the effect of both hydrophilic and hydrophobic polymer leading to controlled and prolonged release of drug (Figure 1, DVF5). It seems the pores formed by hydrophobic polymer (were filled by hydrophilic polymer making a well balanced matrix. Enhancement of permeability (HPMC) and retardation of permeation (sodium $\mathrm{CMC}, \mathrm{MCC}$ ) caused controlled and prolonged release of drug. Physical characteristics of these tablets were recorded and tabulated in Table 2.

The finally optimized dosage form did not produce any significant burst effect that indicated a low possibility of dose dependent toxicity (in vivo). Release kinetics was determined by fitting the release data in different established equations (zero order, first order, Higuchi model, Korsemeyer-Peppas equation). Table 3 shows values of regression coefficient, release constant and exponent $\mathrm{n}$. First order release data was not satisfactory. The data suggested that kinetics of drug release of DVF5 was best explained by Korsemeyer-Peppas equation $\left(\mathrm{R}^{2}\right.$ $=0.991, \mathrm{n}=0.60)$. This indicated combined effect of diffusion and erosion mechanism on the release of drug.

The formulations after storing at $25^{\circ} \mathrm{C} / 60 \% \mathrm{RH}$ and $40^{\circ} \mathrm{C} / 75 \%$ RH for 6 months showed no change in physical appearance and very slight change in dissolution pattern. The results of the stability studies indicated that the formulations could provide a minimum shelf life of 2 years.

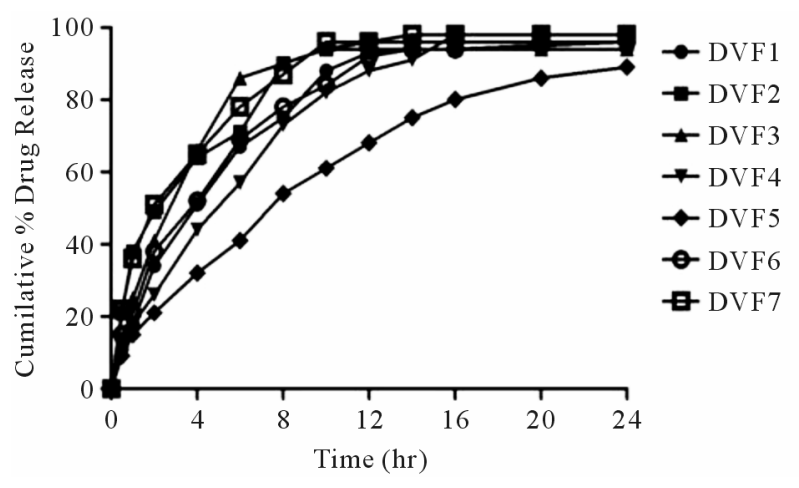

Figure 1. In vitro release profile of Desvenlafaxine_ER tablets. 
Table 2. Drug release kinetic of Desvenlafaxine succinate formulations.

\begin{tabular}{ccccccccc}
\hline & & DVF1 & DVF2 & DVF3 & DVF4 & DVF5 & DVF6 & DVF7 \\
\hline $\begin{array}{c}\text { Higuchi model } \\
(\mathrm{M}=\mathrm{k} \sqrt{\mathrm{t}})\end{array}$ & $\mathrm{K}$ & $11.10 \pm 0.84$ & $10.54 \pm 0.445$ & $11.24 \pm 0.713$ & $11.09 \pm 1.130$ & $9.089 \pm 1.20$ & $10.85 \pm 0.736$ & $10.75 \pm 0.349$ \\
& $\mathrm{R}^{2}$ & 0.9403 & 0.9807 & 0.9575 & 0.8976 & 0.8392 & 0.9517 & 0.9885 \\
$\begin{array}{c}\text { Peppas model } \\
(\mathrm{M}=\mathrm{ktn})\end{array}$ & $\mathrm{n}$ & $0.5190 \pm 0.049$ & $0.315 \pm 0.028$ & $0.411 \pm 0.056$ & $0.5794 \pm 0.035$ & $0.6017 \pm 0.018$ & $0.4769 \pm 0.041$ & $0.3256 \pm 0.029$ \\
& $\mathrm{R}^{2}$ & 0.9244 & 0.9314 & 0.855 & 0.9672 & 0.9916 & 0.9373 & 0.9328 \\
$\begin{array}{c}\text { First order } \\
(\mathrm{lnM}=\mathrm{kt})\end{array}$ & $\mathrm{K}$ & 0.1398 & 0.1534 & 0.1323 & 0.1873 & 0.0941 & 0.1482 & 0.1887 \\
& $\mathrm{R}^{2}$ & 0.8745 & 0.8269 & 0.7549 & 0.9481 & 0.9961 & 0.9379 & 0.8811 \\
$\begin{array}{c}\text { Zero order } \\
(\mathrm{M}=\mathrm{kt})\end{array}$ & $\mathrm{K}$ & $3.968 \pm 0.67$ & $3.483 \pm 0.730$ & $3.632 \pm 0.84$ & $4.269 \pm 0.56$ & $3.797 \pm 0.33$ & $3.877 \pm 0.64$ & $3.548 \pm 0.73$ \\
\hline
\end{tabular}

Next, the optimized tablets were intended for its in vivo test in rabbit. Plasma concentration and pharmacokinetic parameters after oral administration of formulated ER matrix tablet DVF5 and Desvenlafaxine suspension were summarized in Figure 2 and Table 3. No sustained blood level of DV was evident after oral administration of the immediate release formulation. No sustained blood level of DV was evident after oral administration of the immediate release formulation. The formulated matrix Tablet (DVF5) showed significantly lowerC $\mathrm{C}_{\max }$ than the immediate release formulation $(\mathrm{P}<$ 0.05 ) and required more time to reach $\mathrm{C}_{\max }\left(\mathrm{t}_{\max }\right.$ is $4 \mathrm{hr}$ ) as compared with immediate release formulation $\left(\mathrm{t}_{\max }\right.$ is 0.5 $\mathrm{hr})$. The AUC increased from $13190.25 \mathrm{hr} * \mathrm{ng} / \mathrm{ml}$ to $35396.83 \mathrm{hr} * \mathrm{ng} / \mathrm{ml}$ for ER tablets. Values of $\mathrm{C}_{\max }$ and $\mathrm{t}_{\max }$ clearly indicated that thedrug release was sustained to about $24 \mathrm{~h}$ after oral administration in rabbits $(\mathrm{n}=3)$. DVF5 Tablets maintained constant plasma concentration up to about $24 \mathrm{~h}$. The maintenance of plasma concentration for longer duration after administration of new formulation (DVF5) indicates its smooth and extended in vivo absorption.

Results of the present study demonstrated that appropriate combination of both hydrophilic and hydrophobic polymers can be successfully employed for formulating extended release tablet of DV. By spray dried granules with PVP. Moreover extended release granules have a unique advantage of lessening chance of dose dumping which is a major problem when highly water-soluble drug is formulated as simple matrix tablets. The investigated sustained release matrix granules in tablet were capable of maintaining constant plasma level of DV up to $24 \mathrm{~h}$ in rabbits. Similar pharmacokinetic results can be predicted in humans also.

\section{Conclusion}

A new sustained release formulation of Desvenlafaxine

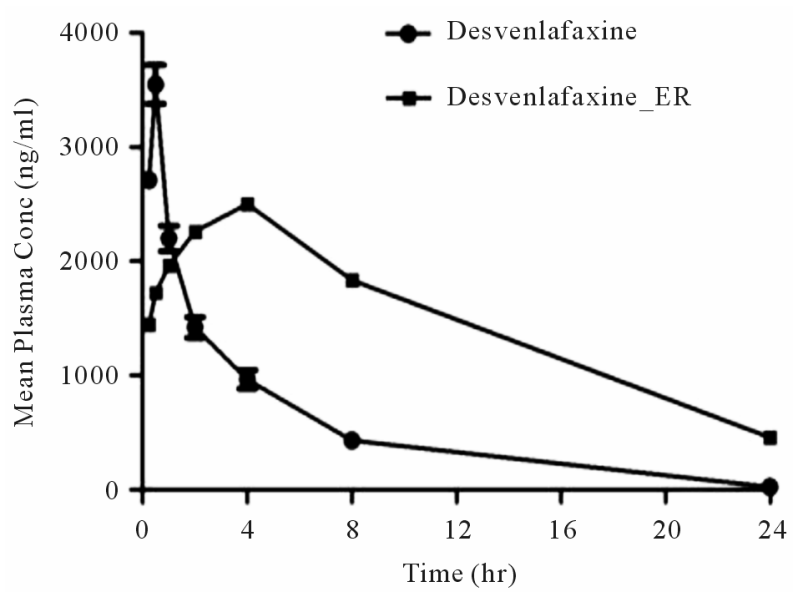

Figure 2. Profile shows mean plasma concentration of Desvenlafaxine against time, following oral administration of Suspension and Tablets (DVF5) to rabbits. Data are represented as mean \pm SD $(n=3)$.

Table 3. Mean $( \pm S D)$ pharmacokinetic parameters of Desvenlafaxine succinate in Rabbits $(n=3)$ orally administered with suspension (50 mg) and ER tablets DVF5 (50 mg).

\begin{tabular}{ccc}
\hline PK Parameters & Desvenlafaxine & $\begin{array}{c}\text { Desvenlafaxine_ER } \\
\text { (DVF5) }\end{array}$ \\
\hline Cmax (ng/mL) & $3550 \pm 294.3$ & $2500 \pm 62$ \\
Tmax (hr) & 0.5 & 4 \\
AUClast $(\mathrm{hr} * \mathrm{ng} / \mathrm{mL})$ & $13190.25 \pm 1829.1$ & $35396.83 \pm 1253.62$ \\
\hline
\end{tabular}

succinate monohydrate has been developed and evaluated for its in vitro and in vivo drug release. Extended release granules in the form of matrix tablet were found to be an effective technique for a highly water-soluble drug Desvenlafaxine succinate monohydrate. Bioavailability studies should be done to assess the usefulness of this formulation in comparison with existing market products (Pristq) of extended release Desvenlafaxine 
succinate formulations on healthy human volunteers.

\section{REFERENCES}

[1] P. D. Alfinito, C. Huseltona, X. H. Chena and D. Deecher, "Pharmacokinetic and Pharmacodynamic Profiles of the Novel Serotonin and nor Epinephrine Reuptake Inhibitor Desvenlafaxine Succinate in Ovariectomized SpragueDawley Rats," Brain Research, Vol. 1098, 2006, pp. 7178.

[2] D. Z. Lieberman and S. H. Massey, "Desvenlafaxine in Major Depressive Disorder: An Evidence-Based Review of Its Place in Therapy," Core Evidence, Vol. 4, 2009, pp. 67-82.

[3] B. N. Singh, "Modified-Release Solid Formulations for Colonic Delivery," Recent Patents on Drug Delivery \& Formulation, Vol. 1, No. 1, 2007, pp. 53-63. doi: $10.2174 / 187221107779814122$

[4] R. Perry, "Desvenlafaxine: A New Seratonin-Norepinephrine reuptake Inhibitor for the Treatment of Adults with Major Depressive Disorder," Clinical Therapeutics, Vol. 31, Part 1, 2009, pp. 1374-1404. doi:10.1016/j.clinthera.2009.07.012,

[5] G. Völgyi and E. Baka, "Study of pH-Dependent Solubility of Organic Bases. Revisit of Henderson-Hasselbalch relationship," Analytica Chimica Acta, Vol. 673, No. 1,
2010, pp. 40-46. doi:10.1016/j.aca.2010.05.022

[6] M. J. Zou and Y. Wang, "Wax-Matrix Tablet for TimeDependent Colon-SpecificDelivery System of Sophora Flavescens Aiton: Preparationand in Vivo Evaluation," Drug Development and Industrial Pharmacy, Vol. 35, No. 2, 2009, pp. 224-233. doi:10.1080/03639040802258854

[7] M. S. Islam, "In Vitro Release Kinetics Study of Diltiazem Hydrochloride from Wax and Kollidon SR Based Matrix Tablets," Iranian Journal of Pharmaceutical Research, Vol. 7, No. 2, 2008, pp. 101-108.

[8] S. B. Tiwari, T. K. Murthy, R. M. Pai, P. R. Mehta andP. B. Chowdary, "Controlled Release Formulation of Tramadol Hydrochloride Using Hydrophilic and Hydrophobic Matrix System," AAPS PharmSciTech, Vol. 4, No. 3, 2003, pp. 18-23. doi:10.1208/pt040331

[9] F. Taneri, I. Ozcan, and T. Guneri, "In Vitro and in Vivo Evaluation of Oral Tablet Formulations Prepared with Ketoconazole and Hydroxypropyl- $\beta$-cyclodextrin," Drug Delivery, Vol. 17, No. 3, 2010, pp. 152-157.

[10] T. Ishikawa and Y. Watanabe, "Effect of Hydroxypropylmethylcellulose (HPMC) on Therelease Profiles and Bioavailability of a Poorly Water-Solubledrug from Tablets Prepared Using Macrogol and HPMC," International Journal of Pharmaceutics, Vol. 202, No. 1, 2000, pp. 173-178. doi:10.1016/S0378-5173(00)00426-9 